\title{
Human-to-dog Transmission of SARS-CoV-2 Lota Variant: Should COVID-19 Patients Avoid Close Contact with their Pets During Illness?
}

Ricardo Rivero

University of Córdoba

Evelin Garay

University of Córdoba

Yesica Botero

University of Córdoba

Hector Serrano-Coll

University of Córdoba

Bertha Gastelbondo

University of Córdoba

Marina Muñoz

Universidad del Rosario

Nathalia Ballesteros

Universidad del Rosario

Sergio Castañeda

Universidad del Rosario

Luz Patiño

Universidad del Rosario

Juan David Ramirez

Universidad del Rosario

Alfonso Calderon

University of Córdoba

Camilo Guzman

University of Córdoba

Caty Martinez-Bravo

University of Córdoba

Ader Aleman

University of Córdoba

German Arrieta

Clinica Salud Social

Salim Mattar ( $\nabla$ smattar@correo.unicordoba.edu.co ) 
University of Córdoba

\section{Research Article}

Keywords: Human-to-dog transmission, SARS-CoV-2 lota variant, COVID-19

Posted Date: September 7th, 2021

DOI: https://doi.org/10.21203/rs.3.rs-821033/v1

License: (c) (1) This work is licensed under a Creative Commons Attribution 4.0 International License. Read Full License 


\section{Abstract}

Severe Acute Respiratory Syndrome Coronavirus 2 (SARS-CoV-2), the etiological agent of the current COVID-19 pandemic, has been proven to have a broad range of host species, including non-human primates, as well as domestic animals and pets. A high conservation degree in the structure of the ACE2 receptor, which is the critical protein for viral infection among vertebrate species, could lead to anthroponotic events. The present paper reports the first symptomatic human-to-dog transmission event of the SARS-CoV-2 lota variant in Latin America. We found a total of 21 mutations shared across the complete genomes of owner and dog viral sequences. Further phylogenetic and molecular analysis showed a $100 \%$ support of clade co-localization supporting the transmission event. Spike protein structure prediction of sequenced virus and docking analyses showed that E484K mutation in the receptor-binding domain (RBD) could enhance viral affinity towards dACE2. Therefore, close contact should be avoided between humans infected with SARS-CoV-2 and pets to avoid the appearance of novel mutations of importance in public health from anthroponotic events.

\section{Introduction.}

Severe Acute Respiratory Syndrome coronavirus 2 (SARS-CoV-2), the etiological agent of the current COVID-19 pandemic is a novel virus belonging to the Coronavirus genus and it is genetically closer to bat coronaviruses than human SARS. For the reasons mentioned above, it is known as a viral zoonosis ${ }^{1,2}$. Research on transmission mechanisms of the viruses focuses on person-to-person contact. However, domestic animals' susceptibility to this virus is still uncertain ${ }^{3}$. To date, 11 complete SARS-CoV-2 genomes isolated from dogs have been reported in the Global Initiative on Sharing All Influenza Data (GISAID), from oropharyngeal samples ${ }^{4}$. The mechanism for viral infections depends on the binding between SARS-CoV-2 S Protein Receptor Binding Domain (RDB) and angiotensin-converting enzyme 2 (ACE2) receptor, which is crucial for infection since it allows the internalization of the virion into host cells

${ }^{5}$. Recently, several SARS-CoV-2 variants have emerged with an enhanced affinity towards human ACE2 ${ }^{6}$. Considering that ACE2 receptors are present in several animal species, interspecies infections could arise from human-to-animal contact. However, the efficacy of the cellular union depends on the affinity of the viral RBD towards the host's receptors ${ }^{7,8}$. Recently, several variants of SARS-CoV-2 have emerged with an augmented infectious capacity and neutralization-escape ability, these variants carry mutations in the spike protein such as N501Y, E484K, and K417T which have been described to have a relationship with higher transmissibility and resistance toward natural-induced and vaccine-elicited neutralizing antibodies 9-11. Recently in February 2021, a novel lineage identified as B.1.526 was reported in $32 \%$ of sequenced genomes in New York and rapidly replaced non-variant lineages. Due to its rapid augment in prevalence and the identification of characteristic mutations in Spike's N-terminal Domain (NTD), Receptor Binding Domain (RBD) and S1/S2 accounting for increased transmissibility, drug-resistance and antibody escape, it has been classified by World Health Organization as a variant of interest, receiving the name lota. The B.1.526 lineage is now circulating in 18 countries, including Colombia, and continues to pose a threat to public health and vaccine efficacy ${ }^{12-14}$. It is still unknown if the emergence of novel variants of concern 
and interest with augmented transmissibility account for enhanced infectivity of non-human hosts. Research of a more significant scale under the "One Health" approach is needed to assess the feasibility of direct human-to-animal transmission of SARS-CoV-2 in domestic environments to understand better the dynamics of the viral infections and their risk towards other species, including humans. This research performs viral genome analysis through next-generation sequencing of two SARS-CoV-2 clinical isolates from a dog and its owner. Given the relevance of this issue, this research aimed to perform a molecular, phylogenetic, and molecular docking approach of a case of SARS-CoV-2 lota variant human-to-dog transmission in Colombia

\section{Results.}

\section{Clinical case description of the infected canine.}

A 54-year-old female presenting moderate clinical manifestations of COVID-19, such as fever, cough, headache, dyspnea, chest pain, and compromise of the lung parenchyma, tested positive for SARS-CoV-2 by RT-qPCR on April 19th, 2021, with a $\mathrm{Ct}=15.13$. Her two years old German shepherd dog presented sneezing, cough, hyaline rhinorrhea, diarrhea, vomiting, adynamia, and lack of appetite for three days after having frequent close contact with its unvaccinated owner, which was in knowledge of the research being done by Universidad de Cordoba and reached out to researchers for pet's sample collection. RTqPCR was conducted from oropharyngeal and rectal swab samples collected from the dog, resulting in a positive SARS-CoV-2 diagnostic by RT-qPCR $(\mathrm{Ct}=31.36)$ on May 5th, 2021. A week later, a follow-up RTqPCR test was conducted with a negative result in oropharyngeal and rectal swab samples. The animal did not show any other sign of illness after the negative result (Fig. 1). It is essential to mention that the dog had a strict indoor lifestyle and does not live with other pets. From the 290 domestic animals evaluated in this research study, $87.6 \%$ were felines and $12.4 \%$ were canines. RT-qPCR detected SARSCoV-2 in a cat (EPI_ISL2339859.2) and a dog for total active infection rate of $0.69 \%$.

\section{Molecular and phylogenetic characteristics of viral genomes.}

Two full-genome sequences labeled anonymously as U118 and U117 were generated from RNA samples of an infected dog and its owner. The resulting SARS-CoV-2 genomes were compared with the reference NC_045512.2-Wuhan-Hu-1, lineage assignment in Pango Lineages ${ }^{15}$ identified both sequences as part of B.1.526 lineage, known as lota Variant which is located within the $20 \mathrm{C}$ clade and more specifically within the E484K sub-clade ${ }^{16}$ (Fig. 2). These genomes shared significant mutations and deletions in $M, N$, ORF1a, ORF1b, ORF3a and S. lota's lineage defining T95I mutation was found in the viral spike protein among others such as E484K, D614G, N440K, as well as $\Delta \mathrm{H} 699-\mathrm{V} 70$ and $\Delta \mathrm{Y} 144$ deletions (Table 1), Phylogenetic analysis by maximum-likelihood co-located both sequences within a sub-clade with a support of 100 alongside Colombian B.1.526 sequences (Fig. 2), and Twenty-one out of 25 mutations 
found in U118 were also found within U117 viral genome. Thus, it was confirming the transmission event between the human and its dog.

Table 1

Details of the SARS-Cov-2 genomes sequenced.

\begin{tabular}{|c|c|c|c|}
\hline $\begin{array}{l}\text { Host } \\
\text { (species) }\end{array}$ & ID & Lineage & Mutations observed* \\
\hline $\begin{array}{l}\text { Canis } \\
\text { lupus } \\
\text { familiaris }\end{array}$ & U117 & B.1.526 & $\begin{array}{l}(\mathrm{n}=21) \text { - M (I82T), N (M210I), M (234I); ORF1a (V86F), ORF1a } \\
\text { (L681F), ORF1a (K1319E), ORF1a (T1822I), ORF1a (P2046S), ORF1a } \\
\text { (K3353R), ORF1a (P3504S), ORF1a ( } \text { S3675-F3677); ORF1b } \\
\text { (K82R9), ORF1a (P314L), ORF1a (T1076I); ORF3a (Q57H); S (F157L) } \\
\text { S (N440K), S (E484K), S (D614G), S (D950N9), S (V1228L). }\end{array}$ \\
\hline \multirow[t]{2}{*}{$\begin{array}{l}\text { Homo } \\
\text { sapiens } \\
\text { sapiens }\end{array}$} & U118 & B.1.526 & $\begin{array}{l}(\mathrm{n}=25)-\mathrm{M}(\mathrm{I82T}) ; \mathrm{N}(\mathrm{M} 234 \mathrm{I}) ; \text { ORF1a (V86F), ORF1a (L681F), } \\
\text { ORF1a (K1319E), ORF1a (T1822I), ORF1a (P2046S), ORF1a } \\
\text { (G2509D), ORF1a (K3353R), ORF1a (P3504S), ORF1a ( } \mathrm{S} 3675- \\
\text { F3677); ORF1b (K82R), ORF1b (P314L), ORF1b }\end{array}$ \\
\hline & & & $\begin{array}{l}\text { (T1076I); ORF3a (Q57H); ORF7a (I110V); S (T95I), S (F157L), S } \\
\text { (N440K), S (E484K), S (D614G), S (D950N), S (V1228L), S ( } \Delta \text { H69- } \\
\text { V70), S ( } 4 \text { Y144). }\end{array}$ \\
\hline
\end{tabular}

*In parentheses: amino acid substitutions/position aligned with reference NC_045512.2- Wuhan-Hu-1.

$\mathrm{N}$ : nucleoprotein; M: membrane glycoprotein M; ORF: open reading frame; S: spike protein.

\section{Predicted structure of the isolated SARS-CoV-2 Spike protein and molecular docking of RBD and dACE2.}

Predicted 3D structures were generated from the sequence U118 for N-terminal domain, Receptor Binding Domain and S2 of the lota S protein (Fig. 3). Docking analyses for lota RBD - dACE2 and wtRBD - dAC2 showed a standard free binding energy $\left(\Delta G^{\circ}\right)$ of -295.58 and $-264.11 \mathrm{~kJ} \mathrm{~mol}^{-1}$ respectively, which suggests a higher affinity of lota's RBD towards dACE2 compared to wild-type SARS-CoV-2 RBD. Also, electrostatic potential mapping evidenced that aminoacid substitutions found in lota's RBD predicted structure accounted for more positively charged residues when compared to wild-type protein, which could play a role in augmenting viral protein's affinity towards the receptor (Fig. 4). Molecular docking analysis between lota RBD and dACE2, identified 27 interface residues within $5 \AA$ distance including N501, K484 and K417 (Fig. 5).

\section{Discussion.}

Our results report the first case of human-to-dog transmission of SARS-CoV-2 in Latin America with successful isolation and sequencing of both viral full-genomes, and show concordance with previously reported studies on SARS-CoV-2 infection in dogs that had prolonged close contact with their owners 3,17 . 
A seroprevalence study carried out in Croatia found a $7.56 \%$ seropositivity in 172 randomly selected dogs living with healthcare, laboratory and veterinary personnel ${ }^{17}$. On the other hand, studies done in Spain and Italy could not obtain positive RT-qPCR from dog samples exhibiting pulmonary complications, but found a seroprevalence of $25 \%$ from dogs living in COVID-19(+) households, this indicates their susceptibility to SARS-CoV-2 infection and its relationship to close-contact with humans rather than dogto-dog 7,18 .

An experimental infection carried by Bosco-Lauth et al ${ }^{8}$ in cats and dogs that were inoculated with $3.0 \mathrm{e} 5$ and $1.45 \mathrm{e} 5 \mathrm{PFU}$ intranasally evidenced viral shedding occurring up to 5 days post-infection (DPI) in cats and moderate ulcerative, suppurative lympho- plasmocytic rhinitis in the nasal turbinates along with mild lymphoplasmacytic tracheitis but showed no signs of clinical disease, no weight-loss and body temperature $<39.5^{\circ} \mathrm{C}$, viral isolation was accomplished from trachea, nasal turbinates and esophagus of necropsied cats on day five post-infection. Contrastingly, no viral shedding nor clinical signs of disease was found in the experimental group of infected dogs with a seroconversion against RBD at $14 \mathrm{DPI}^{8}$. In our study, despite we did not perform viral isolation from samples, the upper respiratory tract clinical signs of disease observed in the infected dog could suggest active viral replication in lung tissue as well as in intestinal tract evidenced by the appearance of gastrointestinal signs such as vomiting, diarrhea, adynamia and lack of appetite.

The molecular and phylogenetic analyses of the obtained sequences support our hypothesis of a transmission event between the dog and its human owner, with both genomes being located within the same clade with a branch support of 100 and a total of 21 mutations shared between both sequences across isolated viral genomes. It is remarkable that these infections were due to the lota SARS-CoV-2 variant (Lineage B.1.526) which carries several mutations in the spike protein that confers augmented transmissibility and resistance to neutralization by naturally acquired and vaccine-elicited antibodies such as E484K mutation, which has been related to attenuation of anti-RBD neutralization ${ }^{19}$ and N440K has been observed to reduce viral susceptibility (up to 28-fold) to monoclonal antibody-based antiviral treatment ${ }^{14}$. This mutations reduces complementarity and electrostatic affinity between neutralizing antibodies and RBD, which could promote an enhanced viral immune evasion in both humans and animals, as well as an increase in reinfection cases and reduction the efficacy of anti-SARS-CoV-2 vaccines ${ }^{12,20,21}$.

Other spike mutations found in the obtained sequences such as N440K and D614G have been observed to reduce the efficacy of monoclonal antibody-based antiviral treatment up to 28-fold and augment the viral infectivity ${ }^{14,22}$. Furthermore, it is important to emphasize that mutation D164G confers increased transmissibility to SARS-CoV-2 by stabilizing Spike protein, this prevents the S1 and S2 segments to be cleaved before RBD interacts with ACE2 receptor ${ }^{23}$. This could be associated with the greater affinity observed by Zhang et al ${ }^{24}$ towards dog ACE2 (dACE2) of variants carrying D614G mutation when compared to Wu-1 SARS-CoV-2. Therefore, mutation D614G could play a key role for anthropozoonotic transmission of SARS-CoV-2 ${ }^{24}$. 
In the other hand, in vitro studies have reported that N501Y mutation found in several variants such as Alpha (B.1.1.7), Gamma (P.1) and Delta (B.1.617.2) enhances the affinity of RBD towards dACE2, accounting for a 3 -fold increase in affinity $\left(K_{D}=37.1\right)$. Hence, wild-type RBD $\left(K_{D}=123 n M\right)$, augmented affinity and higher viral loads found in lineages carrying D614G mutation that was found in the present study, and it could cause an increase in human-to-animal transmission 22,24 .

Zooanthroponosis has occurred in a mink farm in Denmark. this led to the culling of more than 17 million animals, and the emergence of a novel SARS-CoV-2 lineage with reduced susceptibility to antibodyneutralization (Mink cluster $V$ ) ${ }^{25-28}$. Due to the wide host range of SARS-CoV-2, self-isolation measures should be taken by humans to avoid the occurrence of zooanthroponosis events and public health risks which could lead to the emergence of novel lineages with new mutations of importance ${ }^{29}$.

Our study shows in silico that these mutations could account for an increase in the risk of zooanthroponosis events. However, in vitro experiments should be carried out for determining the affinity of VOC and VOI RBD towards animal hosts in order to assess the impact of the emergence of these novel and more transmissible lineages in non-human hosts.

In conclusion, the present study was the first reported case of a human-to-dog transmission event in Latin America supported by the sequencing and molecular characterization of both viral genomes. The identification of the lota variant of interest as the causative agent of this infection adds to the discussion on the importance of self-isolation measures between infected humans and pets. Considering the emergence of novel and more transmissible variants of SARS-CoV-2 could have an enhanced capability of infecting non-human hosts. Epidemiological and molecular surveillance of zooanthroponosis in pets co-habiting with SARS-CoV-2 patients is mandatory since it could represent an animal mutations risk for public health.

\section{Methods.}

\section{Sample collection and detection of SARS-CoV-2.}

Two hundred ninety samples were collected from domestic animals in 8 municipalities of the Cordoba department located in the north-western Colombian Caribbean, informed consent was obtained from humans and animal legal guardians for sample collection in this study. Oropharyngeal and rectal swabs were collected for felines and canines as well as nasopharyngeal samples for humans as a part of the genomic and epidemiologic surveillance of pets living in COVID-19(+) patients' households. Samples were conserved in a Viral Transport medium for SARS-CoV-2 detection by RT-qPCR. Briefly, RNA extraction was carried out using Thermofisher ${ }^{\text {TM }}$ Genejet viral RNA/DNA extraction kit by following the manufacturer's instructions for swab samples. Then, purified RNA was tested by RT-qPCR detection of SARS-CoV-2 E Gene following Charité Berlin protocol ${ }^{30}$. Those animal samples with a Ct value $\leq 32$ was then subjected to next-generation sequencing of the virus' complete genome. Sample collection was approved by Universidad de Cordoba's Veterinary Medicine faculty and its Ethics Committee number 005 
(May 26th, 2021). According to national and international guidelines, including Law 84 of 1989 of the Congress of the Republic of Colombia, national guidelines of animal protection, resolution 8430 of Colombia's Health Ministry and articles 87 and 88 of the 1989's Universal declaration of Animal Rights. Additionally, animals and humans were sampled in compliance of CDC's Guidelines for Safe Work Practices in Human

and Animal Medical Diagnostic Laboratories and WHO Laboratory Biosafety Manual, ensuring human and animal wellbeing ${ }^{31,32}$.

\section{Sequencing of RT-qPCR positive samples.}

Samples were subjected to whole-genome sequencing. Sequence libraries were prepared from RNA extracted from each nasopharyngeal/oropharyngeal swab per individual using the ARTIC Network protocol (https://artic.network/ncov-2019). Long-read Oxford Nanopore MinION sequencing was performed with the MinKNOW application (v1.5.5). Initially, raw Fast5 files were base called, and demultiplexed using Guppy; subsequently, reads were filtered by quality and length, eliminating possible chimeric and low-quality reads. Finally, genome assemblies were obtained following the MinION pipeline described in the ARTIC bioinformatics pipeline (https://artic.network/ncov-2019/ncov2019-

bioinformatics-sop.html). Each assembly was typed based on the PANGOLIN nomenclature lineage allocator and SNPs identification was performed using the clade assignment, mutation calling and sequence quality checking tool, NextClade, which identifies differences between sequences and a reference sequence used by Nextstrain.

\section{Phylogenetic analysis of the sequences.}

Briefly, two datasets were generated from virus sequences reported in GISAID ${ }^{4,33,34}$. For humans, 1855 viruses have been reported from Colombia in GISAID, incomplete sequences ( $<29000 \mathrm{bp}$ ) and sequences with low coverage $(>5 \% \mathrm{Ns}$ ) were excluded from the analysis resulting in a final dataset of 1002 sequences including the dog and owner. On the other hand, another dataset was created with sequences of SARS-CoV-2 detected in Canis lupus familiaris (dog), Felis catus (cat), Panthera leo (lion), Panthera tigris (tiger) and Neovison vison (mink) reported in GISAID globally by following the selection criteria as mentioned above to evaluate phylogenetic between globally reported sequences from cohabiting animals. The two datasets composed of a total of 1002 and 1057 sequences were subjected to multiple sequence alignment (MSA) against reference sequence NC_04551 in MAFFT v $7.48^{35}$ using "addfragments" ${ }^{35}$ functionality within the webserver. Then, alignments were manually edited in Bioedit v.7.2.5 in order to add sample collection date to FASTA accession IDs as defined by REGEX ( $\backslash d \backslash d \backslash d \backslash d$ $\backslash d \backslash d-\backslash d \backslash d)$. Maximum-likelihood phylogenetic trees were reconstructed for each dataset in IQtree v.2.1.3 ${ }^{36}$, ModelFinder ${ }^{37}$ was run for the datasets to determine the best-fit substitution model, this resulted in $\mathrm{GTR}+\mathrm{F}+\mathrm{l}$ being selected for tree reconstruction, branch support was calculated using UFBoot $2^{38}$ within 
IQTree2. The consensus tree was visualized and tailored in FigTree v.1.4.4 ${ }^{39}$ and rooted to NC_045512. Trees were exported in Newick format and then edited in $\mathrm{ITOL}{ }^{40}$ for the addition of annotation schemes.

\section{Structural and molecular docking analysis of SARS-CoV-2 lota Spike protein.}

Predicted 3D models of spike protein domains, namely Amino-terminal Domain (NTD), Receptor Binding Domain (RBD), and S1/S2 were constructed in AlphaFold $2{ }^{41}$ from the sequence of SARS-CoV-2 lota (B.1.526) retrieved from the infected human, multiple sequence alignment for AlphaFold2 was done in MMseqs $2{ }^{42}$. Then, molecular docking analysis was carried out in HDOCK for comparing the affinity of wild-type SARS-CoV-2 RBD (wtRBD) (Protein Database accession ID 6M0J:B) ${ }^{43}$ and lota RBD towards both Human (hACE2) and dog (dACE2) ${ }^{24}$ angiotensin converting enzyme 2 (6MOJ:A and 7E3J:A respectively). Generated models for each interaction were ranked according to Standard binding free energy $\left(\Delta G^{\circ}\right)^{44}$. Poisson-Boltzmann electrostatics visualizations were generated with PDB2PQR plugin within PyMOL ${ }^{45}$.

\section{Declarations}

\section{Acknowledgements.}

The authors acknowledge the work done by laboratory staff of Instituto de Investigaciones Biologicas del Tropico in SARS-CoV2 detection of the samples. We appreciate the support of dog owner which allowed samples to be collected and this research to be published. Authors greatly acknowledge the originating and submitting laboratories for sharing genetic sequences and associated data through the GISAID initiative for SARS-CoV-2 genome sequences. This research was funded by Colombia's Science Ministry (Minciencias), BPIN 20200000100090.

\section{Author Contributions.}

R.R contributed to the design of the study, protein structure prediction and writing of the manuscript; E.G and R.R contributed by doing phylogenetic analysis and writing; M.M, N.B, S.C, L.H.P and J.D.R contributed to genome isolation, sequencing, assembly, analysis and quality control. Y.B, A.A and C.M.B contributed to sample collection, viral detection and clinical case characterization. R.R, H.S.C, B.G, J.D.R, A.C, C.G, G.A and S.M contributed to data analysis, result discussion, and reviewing of the manuscript. All the authors reviewed the manuscript.

\section{Competing interests.}




\section{Materials and correspondence.}

All material requests and correspondence shall be addressed to Professor Salim Mattar (smattar@correo.unicordoba.edu.co).

\section{References}

1. Reina, J. \& El SARS-CoV-2, una nueva zoonosis pandémica que amenaza al mundo. Vacunas, 21, 17-22 (2020).

2. World Health Organization. WHO Coronavirus (COVID-19) Dashboard. WHO Coronavirus (COVID-19) Dashboard With Vaccination Data. Who 1-5 (2021).

3. Sit, T. H. C. et al. Infection of dogs with SARS-CoV-2. Nature, 586, 776-778 (2020).

4. Elbe, S. \& Buckland-Merrett, G. Data, disease and diplomacy: GISAID's innovative contribution to global health. Glob. Challenges, 1, 33-46 (2017).

5. Premkumar, L. et al. The receptor-binding domain of the viral spike protein is an immunodominant and highly specific target of antibodies in SARS-CoV-2 patients. Sci. Immunol.5, (2020).

6. Bosch, B. J., van der Zee, R., de Haan, C. A. M. \& Rottier, P. J. M. The Coronavirus Spike Protein Is a Class I Virus Fusion Protein: Structural and Functional Characterization of the Fusion Core Complex. J. Virol, 77, 8801-8811 (2003).

7. Goumenou, M., Spandidos, D. A. \& Tsatsakis, A. Possibility of transmission through dogs being a contributing factor to the extreme Covid-19 outbreak in North Italy. Mol. Med. Rep, 21, 2293-2295 (2020).

8. Bosco-Lauth, A. M. et al. Experimental infection of domestic dogs and cats with SARS-CoV-2: Pathogenesis, transmission, and response to reexposure in cats. Proc. Natl. Acad. Sci. U. S. A. 117, 26382-26388(2020).

9. Jangra, S. et al. SARS-CoV-2 spike E484K mutation reduces antibody neutralisation. The Lancet Microbe0, (2021).

10. Wibmer, C. K. et al. SARS-CoV-2 501Y.V2 escapes neutralization by South African COVID-19 donor plasma. Nat. Med, 1-4 https://doi.org/10.1038/s41591-021-01285-x (2021).

11. Focosi, D. \& Maggi, F. Neutralising antibody escape of SARS-CoV-2 spike protein: Risk assessment for antibody-based Covid-19 therapeutics and vaccines. Rev. Med. Virol, https://doi.org/10.1002/rmv.2231 (2021).

12. West, A. P., Barnes, C. O., Yang, Z. \& Bjorkman, P. J. SARS-CoV-2 lineage B.1.526 emerging in the New York region detected by software utility created to query the spike mutational landscape. doi:10.1101/2021.02.14.431043. 
13. Annavajhala, M. K. et al. A Novel and Expanding SARS-CoV-2 Variant, B.1.526, Identified in New York. doi:10.1101/2021.02.23.21252259.

14. Federation Drug American (FDA). Fact Sheet for Health Care Providers Emergency Use Authorization of Bamlanivimab and Etesevimab. 1-36 (2020).

15. O'Toole, Ã. et al. pangolin: lineage assignment in an emerging pandemic as an epidemiological tool. In prep https://academic.oup.com/ve/advance-article/doi/10.1093/ve /veab064/6315289 (2021) doi:10.1093/VE/VEAB064.

16. Rambaut, A. et al. A dynamic nomenclature proposal for SARS-CoV-2 lineages to assist genomic epidemiology. Nat. Microbiol, 5, 1403-1407 (2020).

17. Stevanovic, V. et al. Seroprevalence of SARS-CoV-2 infection among pet animals in Croatia and potential public health impact. Transbound. Emerg. Dis, 00, 1-7 (2020).

18. Perisé-Barrios, A. J. et al. Humoral responses to SARS-CoV-2 by healthy and sick dogs during the COVID-19 pandemic in Spain. Vet. Res. 2021521 52, 1-11 (2021).

19. Jangra, S. et al. SARS-CoV-2 spike E484K mutation reduces antibody neutralisation. The Lancet Microbe, 2, e283-e284 (2021).

20. Cele, S. et al. Escape of SARS-CoV-2 501Y.V2 from neutralization by convalescent plasma. Nat. 2021 5937857, 593, 142-146 (2021).

21. Andreano, E. et al. SARS-CoV-2 escape in vitro from a highly neutralizing COVID-19 convalescent plasma. bioRxiv Prepr. Serv. Biol, https://doi.org/10.1101/2020.12.28.424451 (2020).

22. Fernández, A. Structural Impact of Mutation D614G in SARS-CoV-2 Spike Protein: Enhanced Infectivity and Therapeutic Opportunity.ACS Med. Chem. Lett11, (2020).

23. Zhang, L. et al. SARS-CoV-2 spike-protein D614G mutation increases virion spike density and infectivity. doi:10.1038/s41467-020-19808-4.

24. Zhang, Z. et al. The molecular basis for SARS-CoV-2 binding to dog ACE2. Nat. Commun, 12, 4195 (2021).

25. Gobeil, S. M. C. et al. Effect of natural mutations of SARS-CoV-2 on spike structure, conformation and antigenicity. bioRxiv Prepr. Serv. Biol, https://doi.org/10.1101/2021.03.11.435037 (2021).

26. Mallapaty, S. COVID mink analysis shows mutations are not dangerous - yet. Nature, 587, 340-341 (2020).

27. Oude Munnink, B. B. et al. Transmission of SARS-CoV-2 on mink farms between humans and mink and back to humans.

28. World Health Organization. SARS-CoV-2 mink- associated variant strain - Denmark. https://www.who.int/emergencies/disease-outbreak-news/item/2020-DON301.

29. Luan, J., Lu, Y., Jin, X. \& Zhang, L. Spike protein recognition of mammalian ACE2 predicts the host range and an optimized ACE2 for SARS-CoV-2 infection. (2020) doi:10.1016/j.bbrc.2020.03.047. 
30. Corman, V. et al. Diagnostic detection of 2019-nCoV by real-time RT-PCR. https://virologieccm.charite.de/en/ (2020).

31. Frieden, T. R. et al. Morbidity and Mortality Weekly Report Guidelines for Safe Work Practices in Human and Animal Medical Diagnostic Laboratories Recommendations of a CDC-convened, Biosafety Blue Ribbon Panel Centers for Disease Control and Prevention MMWR Editorial and Production Staff (MMWR Editorial Board, 2012).

32. World Health Organization. Laboratory biosafety manual Third edition. World Health Organization (2004).

33. Shu, Y. \& McCauley, J. G. I. S. A. I. D. Global initiative on sharing all influenza data - from vision to reality. Eurosurveillance, 22, 30494 (2017).

34. GISAID \& Initiative, G. I. S. A. I. D. Adv. Virus Res. 2008, 1-7 (2020).

35. Katoh, K. \& Frith, M. C. Adding unaligned sequences into an existing alignment using MAFFT and LAST., 28, 3144-3146 (2012).

36. Minh, B. Q. et al. IQ-TREE 2: New Models and Efficient Methods for Phylogenetic Inference in the Genomic Era. Mol. Biol. Evol, 37, 1530-1534 (2020).

37. Kalyaanamoorthy, S., Minh, B. Q., Wong, T. K. F., von Haeseler, A. \& Jermiin, L. S. ModelFinder: fast model selection for accurate phylogenetic estimates. Nat. Methods, 2017146 14, 587-589 (2017).

38. Hoang, D. T., Chernomor, O., von Haeseler, A., Minh, B. Q. \& Vinh, L. S. UFBoot2: Improving the Ultrafast Bootstrap Approximation. Mol. Biol. Evol, 35, 518-522 (2018).

39. Rambaut, A. FigTree. http://tree.bio.ed.ac.uk/software/figtree/.

40. Letunic, I. \& Bork, P. Interactive Tree Of Life (iTOL) v5: an online tool for phylogenetic tree display and annotation. Nucleic Acids Res, 49, W293-W296 (2021).

41. Jumper, J. et al. Highly accurate protein structure prediction with AlphaFold. Nat. 2021,1-11 https://doi.org/10.1038/s41586-021-03819-2 (2021).

42. Mirdita, M., Steinegger, M. \& Söding, J. MMseqs2 desktop and local web server app for fast, interactive sequence searches., 35, 2856-2858 (2019).

43. Lan, J. et al. Structure of the SARS-CoV-2 spike receptor-binding domain bound to the ACE2 receptor. Nature, 581, 215-220 (2020).

44. Du, X. et al. Insights into protein-ligand interactions: Mechanisms, models, and methods. International Journal of Molecular Sciencesvol. 17 (2016).

45. Dolinsky, T. J., Nielsen, J. E., McCammon, J. A. \& Baker, N. A. PDB2PQR: an automated pipeline for the setup of Poisson-Boltzmann electrostatics calculations. Nucleic Acids Res, 32, W665-W667 (2004).

\section{Figures}




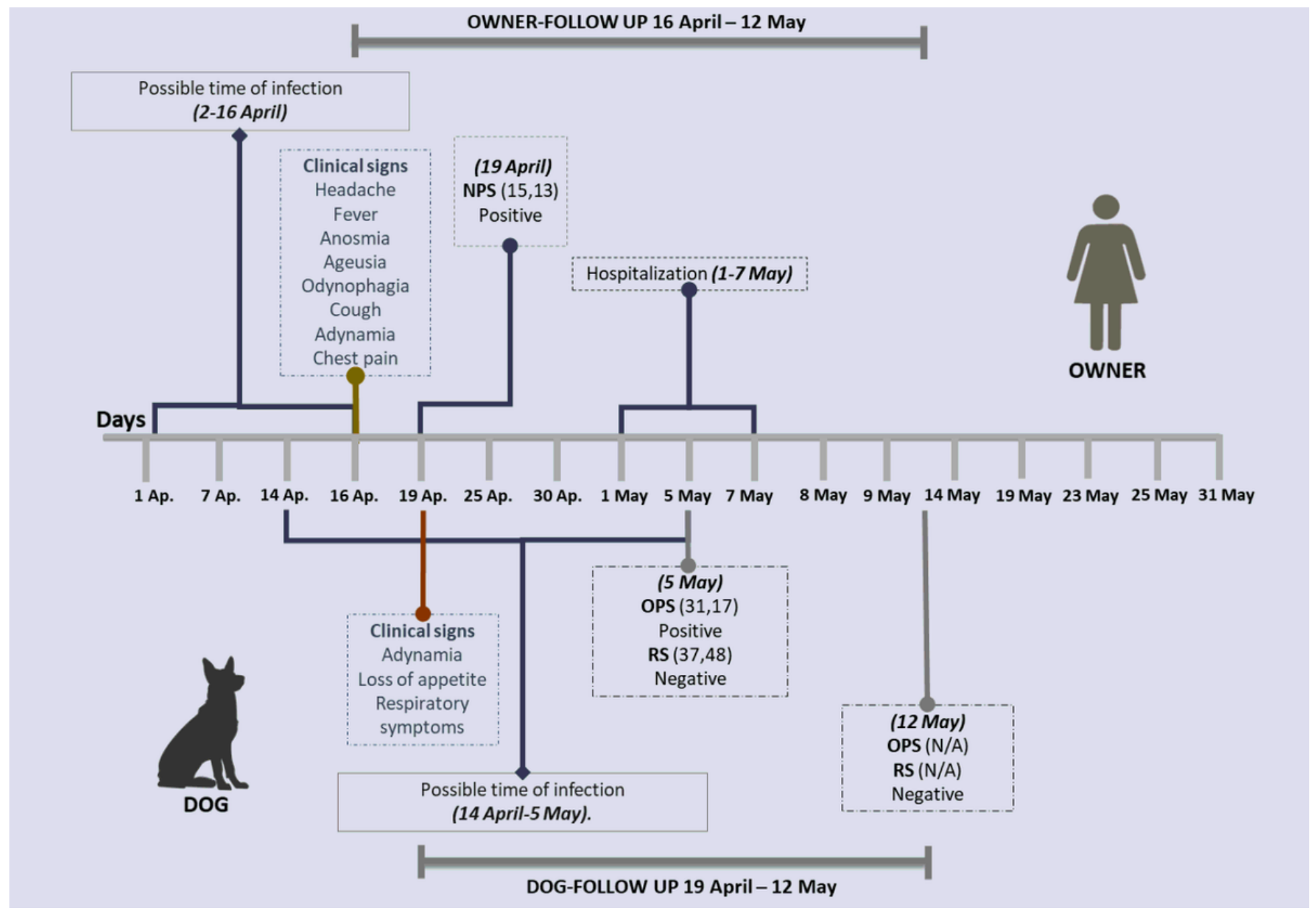

\section{Figure 1}

Timeline of symptom diagnosis, molecular testing, and follow-up.

a.

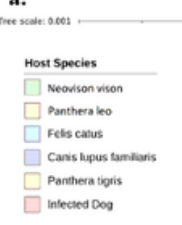

b.

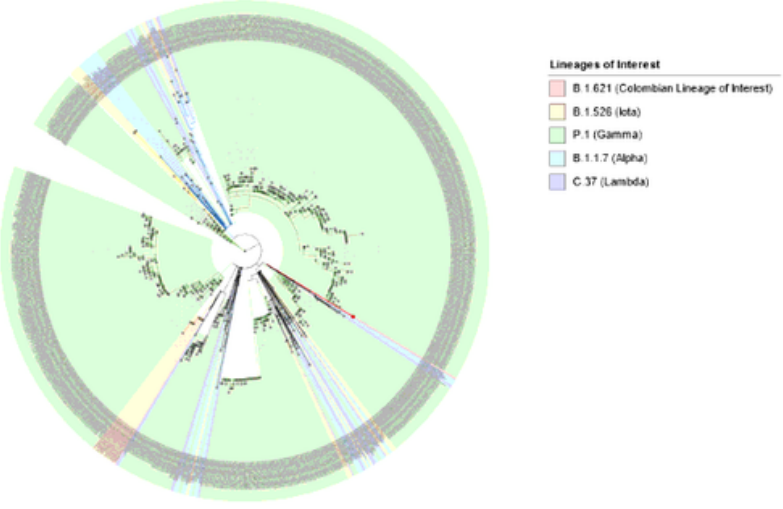

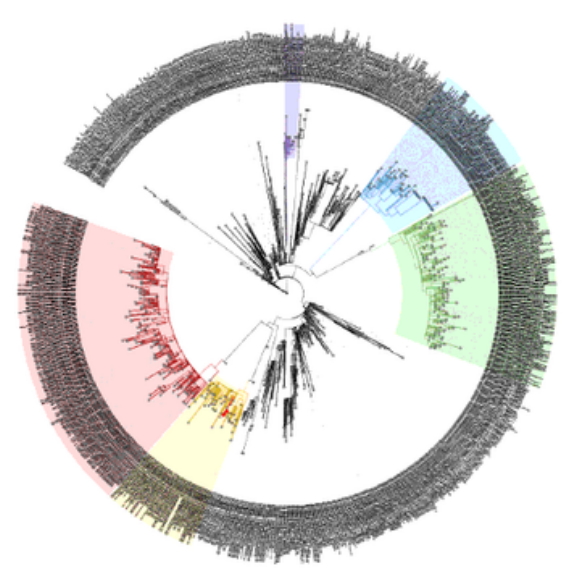

Figure 2 
a) Phylogenetic trees of sequences available in GISAID from minks, lions, tigers, cats, and dogs, U117 is highlighted in red. b) Phylogenetic tree comprising Colombian sequences, VOC, and lineages of interest are annotated within the tree; U117 and human index case U118 are highlighted in red.

a.

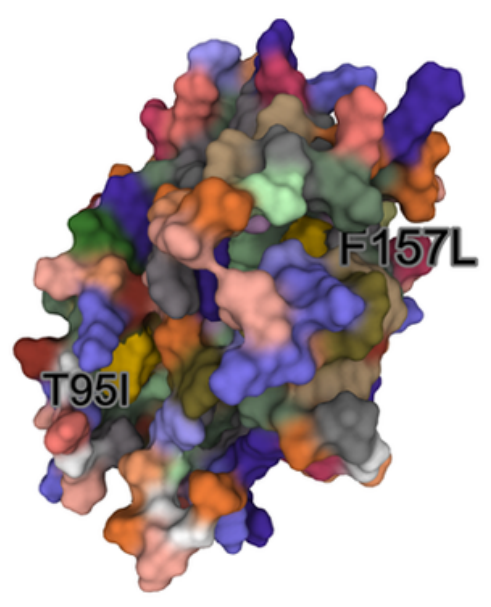

b.

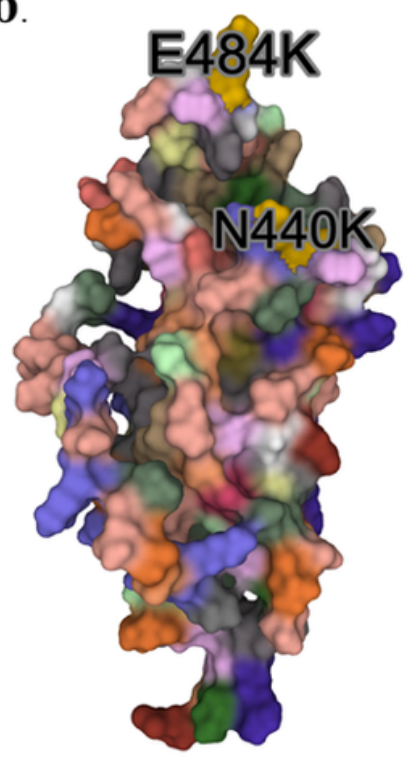

c.

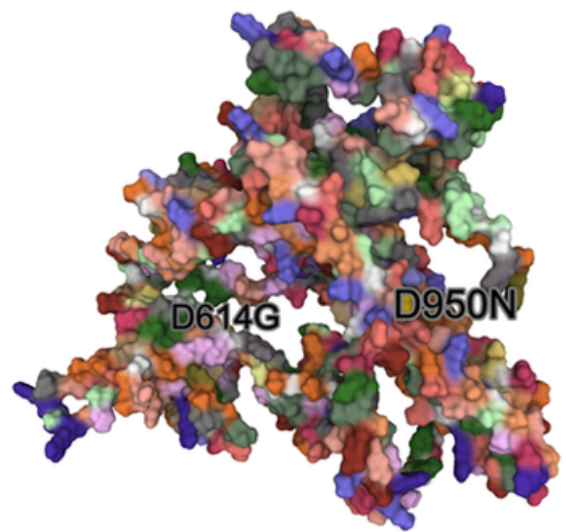

\section{Figure 3}

Predicted structure models of lota SARS-CoV-2 Spike Protein domains (a-c) N-terminal Domain, Receptor Binding Domain, and S1/S2 with mutations highlighted in yellow.

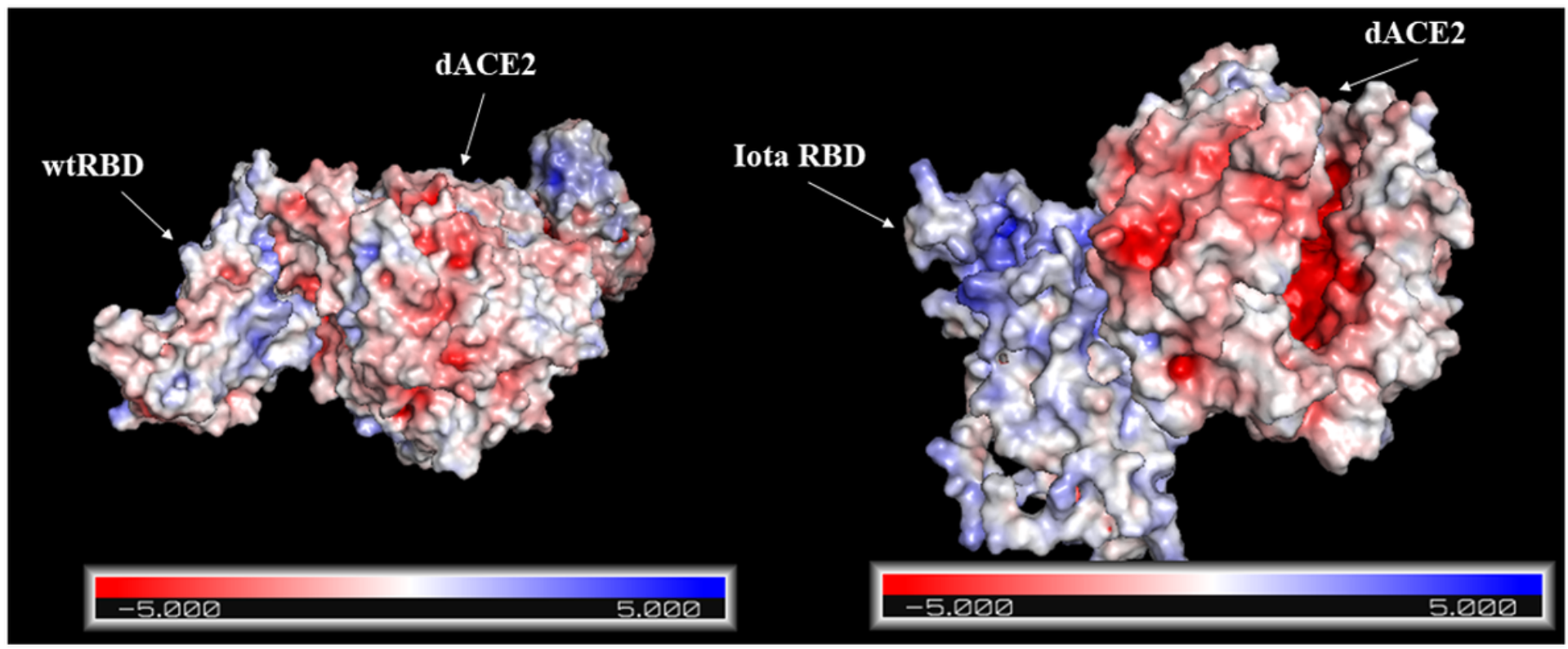

Figure 4

Electrostatic potential map of wtRBD-dACE2 and lota RBD-dACE2. 


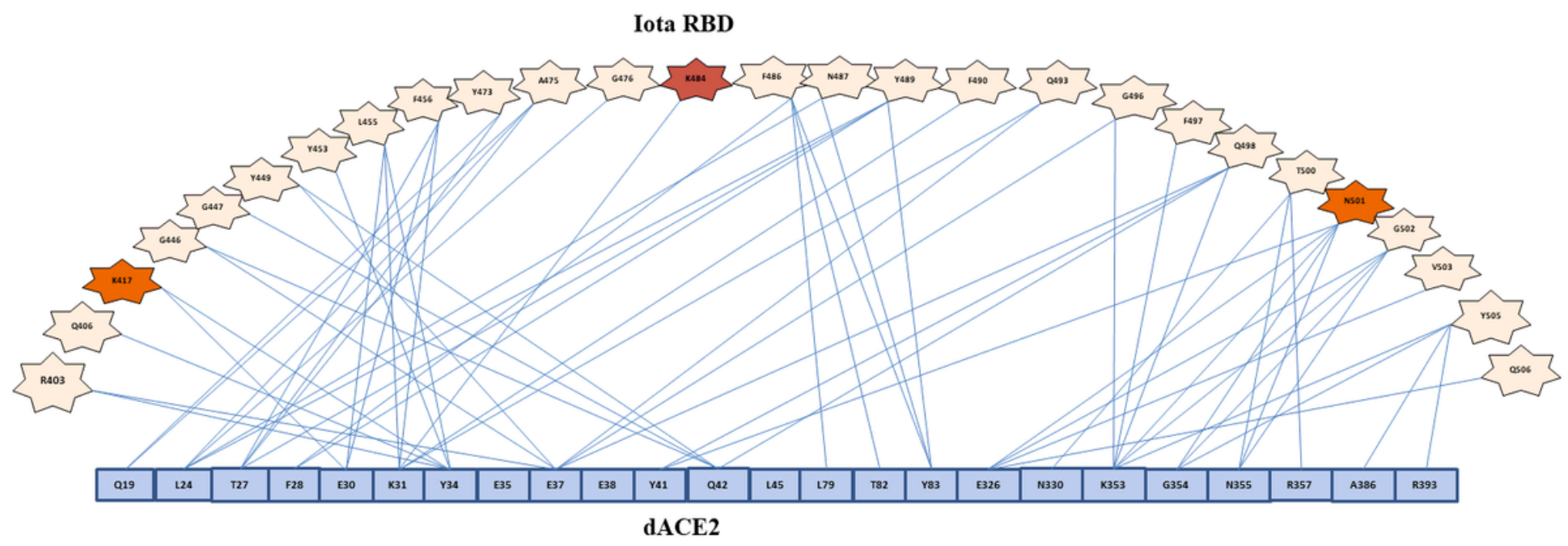

Figure 5

lota SARS-CoV-2 - dACE2 interface residues. Mutation E484K is presented in red; residues 417 and 501 of importance in transmissibility enhancement are highlighted in orange. 